Mon. Not. R. Astron. Soc. 000,111(2002) Printed 1 November $2018 \quad$ (MN IATEX style file v2.2)

\title{
Ionized gas outflow in the isolated S0 galaxy NGC $4460^{\star}$
}

\author{
Alexei Moiseev†, Igor Karachentsev and Serafim Kaisin \\ Special Astrophysical Observatory, Russian Academy of Sciences, Nizhnii Arkhyz, Karachaevo-Cherkesskaya Republic, 369167 Russia
}

Accepted December 29; Received 2009 December 8; in original form 2009 October 1

\begin{abstract}
We use integral-field and long-slit spectroscopy to study the bright extended nebulosity discovered in the isolated lenticular galaxy NGC 4460 during a recent $\mathrm{H} \alpha$ survey of nearby galaxies. An analysis of archival SDSS, GALEX, and HST images indicates that current star formation is entirely concentrated in the central kiloparsec of the galaxy disc. The observed ionized gas parameters (morphology, kinematics and ionization state) can be explained by a gas outflow above the plane of the galaxy caused by a star formation in the circumnuclear region. Galactic wind parameters in NGC 4460: outflow velocity, total kinetic energy - are several times smaller comparing with the known galactic wind in NGC 253, which is explained substantially lower total star formation rate. We discuss the cause of the star formation processes in NGC 4460 and in two other known isolated S0 and E galaxies of the Local volume: NGC 404 and NGC 855. We provide evidence suggesting that feeding of isolated galaxies by intergalactic gas on a cosmological time scale is a steady process without significant variations.
\end{abstract}

Key words: galaxies: elliptical and lenticular - galaxies: ISM - galaxies: kinematics and dynamics - galaxies: starburst - galaxies: individual: NGC 4460

\section{INTRODUCTION}

Isolated elliptical (E) and lenticular ( $\mathrm{S} 0$ ) galaxies are rather rare objects, whose properties differ appreciably from $\mathrm{E}$ and S0 galaxies in clusters and groups. Among the 513 most isolated galaxies of the Local Universe with radial velocities $V_{L G}<3500$ only 19 systems (i.e., about 4 per cent) belong to types E and S0 (Karachentsev et al. 2009). Isolated $\mathrm{E}$ and $\mathrm{S} 0$ galaxies are characterized by low luminosity $\left(M_{B} \simeq-17^{\mathrm{m}} 6\right)$, bluer-than-average colors, and appreciable presence of gas and dust. The nearby lenticular galaxy NGC 404 with $M_{B}=-16.6$, which was found to host an extended HI shell (Del Rio et al 2004) and ultraviolet-bright ring of young stars (Thilker 2009), is a typical example. Among 450 Local-volume (LV) galaxies with heliocentric distances $\mathrm{D}<10 \mathrm{Mpc}$ (Karachentsev et al. 2004) there are only three E and S0 galaxies with absolute magnitudes $\left[-16.0>M_{B}>-18.0\right]$ and negative 'tidal indices' $\mathrm{TI}<0$, which allowed these galaxies NGC 404, NGC 855, and NGC 4460 - to be classified as isolated objects. These objects seem to be local analogues of recently identified by Kannappan, Guie \& Baker (2009)

\footnotetext{
* Based on observations collected with the $6 \mathrm{~m}$ telescope of the Special Astrophysical Observatory of the Russian Academy of Sciences which is operated under the financial support of Science Department of Russia (registration number 01-43)

† moisav@gmail.com
}

'blue-sequence E/S0' galaxies which might form a transition population between late-type spirals/iregulars and earlytype red-sequence galaxies.

An extensive $\mathrm{H} \alpha$ survey of $\mathrm{LV}$ galaxies has been carried out in recent years with the 6-m telescope of the Special Astrophysical Observatory of the Russian Academy of Sciences (SAO RAS) in order to determine rates of star formation in a representative distance-limited sample of galaxies. An analysis of the population of Canes Venatici I scattered cloud of nearby galaxies revealed (Kaisin \& Karachentsev 2008) that the circumnuclear region in NGC 4460 hosts the bright $\mathrm{H} \alpha$ line emission (see Fig. 1), which must be indicative of ongoing star formation. Such a phenomenon appears to be nontypical in an isolated early-type galaxy. This bright emission may be a result of a recent merger of NGC 4460 with a gasrich companion, or of an impact of a massive intergalactic HI cloud onto the central region of NGC 4460. In this case, the $\mathrm{H} \alpha$ nebulosity, which extends along the major axis of the galaxy, may be a product of a recent interaction. An analysis of the kinematics and ionization state of the gas in NGC 4460 helps us understand the nature of this mysterious phenomenon. With this aim in view, we analyzed the available archival images of the galaxy and performed spectroscopic observations of NGC 4460 with the SAO RAS 6-m telescope. Following Tonry et al. (2001), we adopt the distance to the galaxy to be $9.59 \mathrm{Mpc}$ that corresponds to a scale of $46.5 \mathrm{pc} \operatorname{arcsec}^{-1}$. 

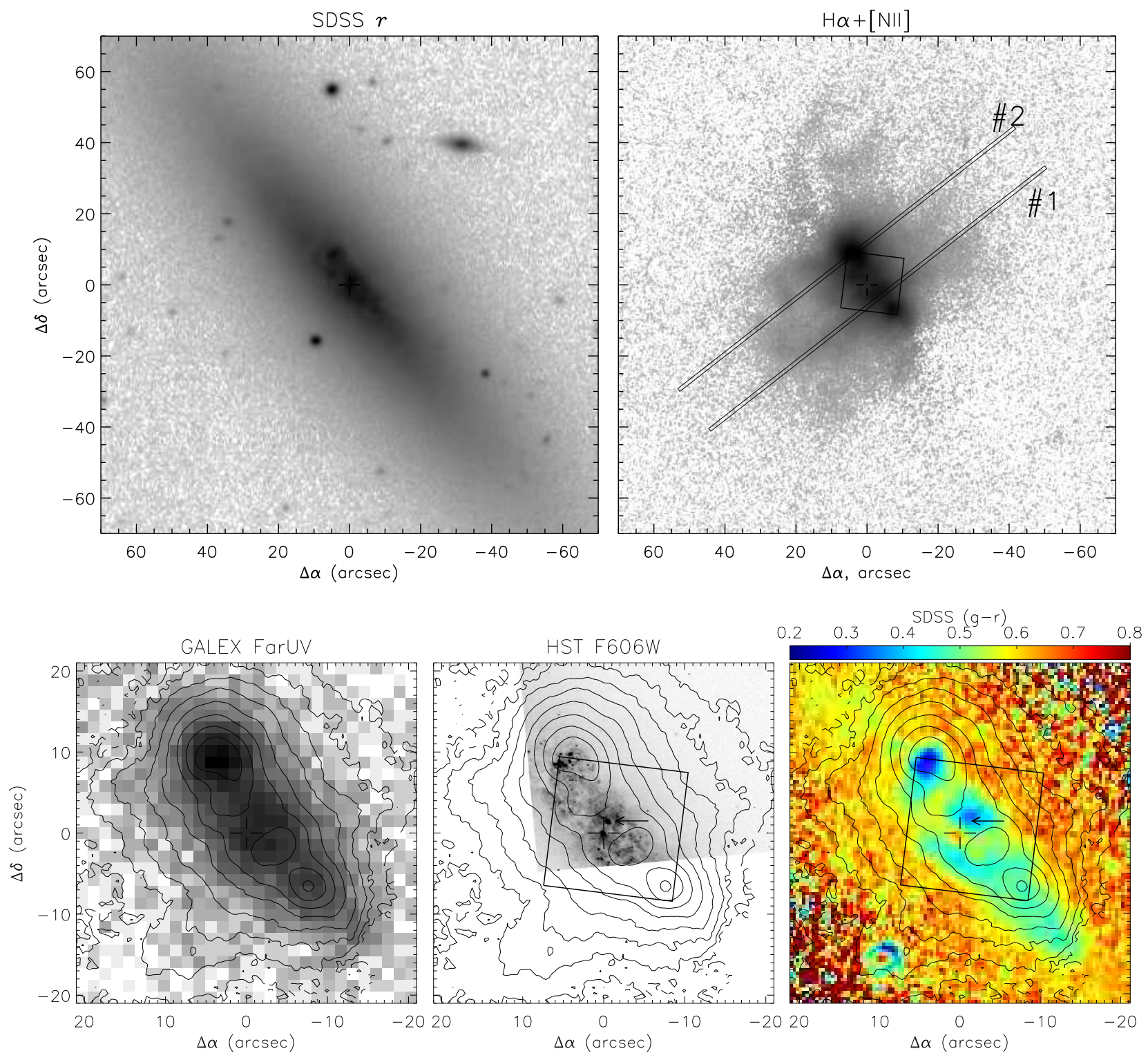

Figure 1. Images of NGC 4460 in logarithmic brightness scale. The upper row shows the SDSS $r$-band image (left) and the H $\alpha+[\mathrm{N}$ II] image (right) taken with the 6-m telescope (Kaisin \& Karachentsev 2008). The locations of the spectrograph slits are shown. Hereafter the black square marks the MPFS field of view. The lower row contains the images of the center of the galaxy with H $\alpha$ line contours superimposed based on GALEX FUV data (left), HST WFPC2/F606W optical map (center) and map of the $(g-r)$ color index according to SDSS (right). The cross marks the galaxy nucleus that is coincided with the kinematic center derived from MPFS data. The arrow shows the position of second circumnuclear knot (see text).

\section{PHOTOMETRIC STRUCTURE OF THE GALAXY}

\subsection{Circumnuclear region}

A comparison of the $\mathrm{H} \alpha+[\mathrm{NII}]$ image of the galaxy with broadband SDSS images (Fig. 11) convincingly demonstrates that most of the emission-line radiation emerges from a compact region in the disc inside the radius $r<20$ arcsec. That is where all HII regions are located, whereas diffuse $\mathrm{H} \alpha+[\mathrm{N}$ II $]$ emission extends along the minor axis of the galaxy on both sides of its nucleus. An analysis of optical and UV images shows that ongoing star formation in
NGC 4460 is concentrated entirely inside the central kiloparsec. First, it follows from the distribution of FAR-UV radiation $(\lambda 1344-1786 \AA)$ according to $G A L E X$ archival data, that is exactly coincident with the circumnuclear HII regions (Fig. 1). Second, the young stellar population found in this region stands out by its bluer color index in SDSS images (see Fig. 1). Star-forming regions make an almost closed ring in the disc of the galaxy. The $r$-band image reveals a compact nucleus with $(g-r)=0.65$, whereas a condensation located 1.5 - 2 arcsec North of the nucleus (marked with arrow on Fig. (1) becomes brighter in blue filters with the color $(g-r)=0.45$. On the HST optical image this 
circumnuclear knot corresponds to compact stellar cluster with a linear size $F W H M=17 \times 10$ pc whose luminosity is only twice lower than that of the compact cluster in the 'main' nucleus. We note that the dynamical center of the gas velocity field (marked with cross on Figs. 1 and 3) coincides with the main nucleus of the galaxy (see below Sect. (5)). The circumnuclear star-forming regions have evidently different evolutionary status, because only $\mathrm{NE}$ of the nucleus does the peak of $\mathrm{H} \alpha$ emission coincide with the 'blue' region on the $(g-r)$ map. At the same time, the two blue regions near the minor axis (including the second nucleus) are unassociated with $\mathrm{H} \alpha+[\mathrm{N}$ II] peaks. These regions must have already lost most of their gas or lack OB stars needed for its ionization.

The SDSS images exhibit individual dust lanes in the inner part of the region. On the HST image these lanes can be easily seen to border HII regions, and they are most probably be associated with dense gas compressed by shocks produced by star-forming processes (SN explosions and winds from massive stars).

\subsection{Orientation of the disc and the brightness distribution}

Using isophotal analysis of SDSS images we found the position angle and ellipticity of the outer $r$ and $i$-band isophotes to be $P A=39.5 \pm 0.4^{\circ}, \epsilon=0.71 \pm 0.02$. According to empirical relation (Hubble 1926), this implies a galaxy inclination of $i=77 \pm 1^{\circ}$. We used the inferred orientation parameters to construct the azimuthally averaged surface-brightness profiles of ubvri SDSS images. In all filters the brightness distribution is almost exactly exponential at $r>30-40$ arcsec, with the brightness excess at $r<20$ arcsec being more conspicuous in blue filters - it is represented by star-forming regions. Figure 2 shows decompositions of the $r$-band surface brightness profile into components. After the subtraction of the outer disc $\left(\mu_{0}=19.4^{m}, h=29.5\right.$ arcsec $)$, brightness excess still remains at $r<30-40$ arcsec. However, it is not a bulge, because after the subtraction of the two-dimensional disc model the brightness contours of the residual image have the same ellipticity as those of the outer disc. The brightness profile at the center can be described fairly well by a second exponential function with almost the same central brightness $\left(\mu_{0}=19.2^{m}\right)$, but with a three times shorter scale length $h=11.5$ arcsec. At the center one can see a low-contrast compact nucleus described above.

It thus follows that only some of the morphological features (smooth outer isophotes, lack of spiral arms) suggest that NGC 4460 may be a lenticular galaxy, whereas the bulge/disc ratio is indicative of a later morphological type, because the spherical component is barely visible in the brightness distribution. Inner exponential discs are often referred to as 'pseudo-bulges' (Kormendy \& Kennicutt 2004). However, the corresponding feature in NGC 4460 it is indeed a bona fide disc whose thickness, according to photometric data, coincides with that of the outer disc.

Multitiered (antitruncation according the classification by Erwin, Beckman \& Pohlen 2005) discs have been found increasingly often in spiral and lenticular galaxies, and they are attributed either to slow internal secular evolution that can only develop in the presence of a bar and/or a spiral (see Kormendy \& Kennicutt 2004, for review), or to a recent minor merging event. Both cases imply the loss of angular mo- mentum by a part of gas moved toward the center, where a burst of star formation currently occurs, and the newly born stars form the inner disc with a short scale length. Recent formation of the inner disc is also evidenced by the fact that its contribution to the combined brightness profile (Fig. 2) is almost everywhere smaller than that of the main disc of the galaxy.

\subsection{Bar or ring?}

In Sect 2.1 we mentioned that the star-forming regions make an almost closed ring in the central kpc. At first sight, the observed distribution of the HII regions has another interpretation, where the two brightest condensations, located almost equidistant from the nucleus $\sim 10$ arcsec to northeast and south-west, belong to the ends of a stellar bar. In this case, the two blue regions appeared on the color-index map (Fig. 1) at $r=2-4^{\prime \prime}$ (included the off-nuclear compact cluster mentioned above) can be considered as part of nuclear compact ring formed on the resonances of this bar.

However, this assumption contradicts to other morphological features. First is a significant asymmetry in the properties of two brightest HII regions. They have different size, $\mathrm{H} \alpha$-luminosity and color index, i.e. different history of their formation. That is strange in the case of lobes on the ends of a bar which should be formed simultaneously. Secondly, the dust in the circumnuclear region has a chaotic distribution (Sect 2.1) instead sharp curved dust lanes expected in a bar. Finally, the isophotal analysis of SDSS red images does not support any significant changes of ellipticity and $P A$ of isophotes at the distances corresponded to the possible bar.

The effect of non-circular gas motions (radial inflow) could be also insufficient in the observed gas velocity field, because the major axis of possible bar coincides with the galaxy line of nodes. Therefore, the regular velocity pattern in the circumnuclear region of ionized gas velocity field (Sect. 5) can not be use as pro or contra arguments about bar. We think that only data on stellar kinematics can evident the existence (or absent) a stellar bar in NGC 4460. However, our analysis of galactic photometry presented above suggests the star-forming pseudo-ring $2 \mathrm{kpc}$ in diameter nested in the inner stellar disc as more reliable description of the observed morphology.

\section{OBSERVATIONS AND DATA REDUCTION}

Long-slit and integral-field (3D) spectral observations were made at the prime focus of the SAO RAS) 6-m telescope. The $2048 \times 2048$ EEV 42-40 CCD was used as a detector in both cases.

The central region of NGC 4460 was observed with the MultiPupil Fiber Spectrograph (MPFS). The MPFS (Afanasiev, Dodonov \& Moiseev 2001) takes simultaneous spectra from 256 spatial elements (constructed in the shape of square lenses) that form on the sky an array of $16 \times 16$ elements ('spaxels') with the angular size $1 \operatorname{arcsec} \operatorname{spaxel}^{-1}$. A bundle of 17 fibers placed at the distance about 3.5 arcmin from the lens array provides the night-sky background spectra simultaneously with objects exposition. The spectral range included bright emission lines of ionized gas: $\mathrm{H} \alpha,[\mathrm{N} \mathrm{II}] \lambda \lambda 6548,6583$ and $[\mathrm{S} \mathrm{II}] \lambda \lambda 6717,6731$. 
Table 1. Log of spectral observations

\begin{tabular}{|c|c|c|c|c|c|c|}
\hline Date & Device & position & $\begin{array}{l}T_{\exp } \\
(\mathrm{s})\end{array}$ & $\begin{array}{l}\text { sp. range } \\
(\AA)\end{array}$ & $\begin{array}{l}\text { sp. resol. } \\
(\AA)\end{array}$ & $\begin{array}{l}\text { seeing } \\
(\text { arcsec })\end{array}$ \\
\hline 27/28 Mar 2007 & MPFS & nucleus & 1800 & $5800-7300$ & 3.5 & 1.7 \\
\hline 15/16 May 2007 & SCORPIO/LS & $\mathrm{PA}=128^{\circ}$, offset 5 arcsec to $\mathrm{SW}$ & 1200 & $6050-7100$ & 2.5 & 1.7 \\
\hline 15/16 May 2007 & SCORPIO/LS & $\mathrm{PA}=128^{\circ}$, offset $9 \operatorname{arcsec}$ to $\mathrm{NE}$ & 900 & $6050-7100$ & 2.5 & 1.8 \\
\hline
\end{tabular}

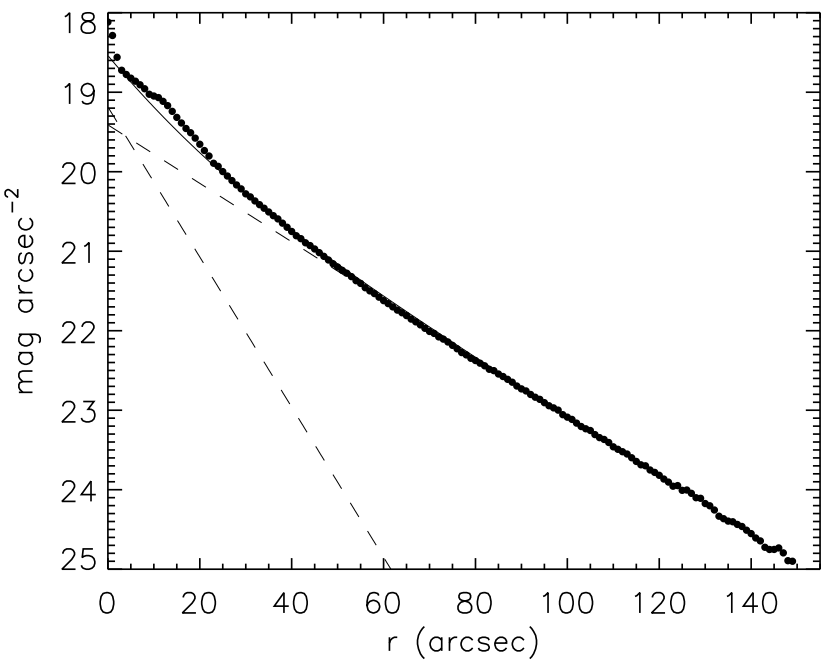

Figure 2. Decomposition of the r-band surface brightness profile (dots) into two discs (the dashed line). The solid line marks the sum of both disc models.

Fig 1 shows the locations of MPFS lens array on the galaxy image, the log of observations is presented in Table 1 . The spectra were reduced using the IDL-based software, the data reduction sequence is briefly described in Moiseev, Valdés \& Chavushyan (2004). The data reduction results in a data cube in which a spectrum corresponds to each image 'spaxel' in two-dimensional field $16 \times 16 \operatorname{arcsec}^{2}$. The data cube was flux-calibrated using the standard star observations taken on the same night, just after the galaxy. The maps and velocity fields of the $\mathrm{H} \alpha,[\mathrm{N}$ II] and [S II] emission lines were constructed using a single-Gaussian fitting.

The long-slit observations have been made with the multi-mode focal reducer SCORPIO Afanasiev \& Moiseev 2005). We obtained two cross-sections in direction near perpendicular to the galactic major axis, along extended $\mathrm{H} \alpha$ filaments and though bright HII-regions in the galaxy disc (hereafter slits \#1 and \#2, see Fig. 1). The slit width was 1 arcsec, the scale along slit was $0.35 \operatorname{arcsec} \mathrm{px}^{-1}$, the $\log$ of observations is given in Table 11. The slit spectra were reduced and calibrated using the IDL-based software, developed in SAO RAS. The parameters of the emission lines (FWHM, flux, velocity) were calculated from a single-Gaussian fitting. A multicomponent structure of the emission line profiles was not detected as well as in MPFS spectra. The long-slit spectra are deeper, because SCORPIO quantum efficiency is significantly higher than MPFS. This fact allows us to detect the weak emission lines $[\mathrm{OI}] \lambda \lambda 6300,6360$ and $\mathrm{He} \mathrm{I} \lambda 6678$ in the brighter HII regions in the galactic disc.

\section{PARAMETERS OF IONIZED GAS}

Fig. 3 shows several maps based on MPFS data. The $\mathrm{H} \alpha$ brightness distribution agrees well with the narrow-band filter image discussed above. The continuum image exhibits a central condensation extending along the N-S direction. Given the lower spatial resolution of the MPFS compared to SDSS data, the condensation must correspond to the double nucleus described in Sect. 2.1 The MPFS field of view contains three bright HII regions. The maps of the $[\mathrm{NII}] / \mathrm{H} \alpha$ and $[\mathrm{SII}] / \mathrm{H} \alpha$ flux ratios indicate a slight enhancement of forbidden-line emission around star-forming regions 1 , whereas the corresponding ratios are minimal at the centers of HI regions (see Fig. 3). In the space between star-forming regions we most likely observe hot gas ejected from these regions and ionized, among other factors, by shocks. The presence of shock fronts around HII regions is further evidenced by dust lines seen at these locations (Sect. 2.1). In these places the $[\mathrm{S} \mathrm{II}] / \mathrm{H} \alpha$ ratio reaches 0.45 , which is close to the usually adopted border between shock ionization and photoionization (see below).

Figure 4 shows variations of the emission-line parameters (flux, radial velocity, $F W H M$ corrected for instrumental broadening) along the slits. The maximum of the emission lines brightness is therefore shifted relative to the peak in brightness of stellar continuum, because bright HII regions in the circumnuclear ring do not lie on the major axis of the galaxy. Beyond the disc, surface brightness in the nebulosity decreases almost exponentially and emission lines can be detected at even greater distance from the disc than on the $\mathrm{H} \alpha+[\mathrm{N} \mathrm{II}]$ image. The $[\mathrm{S} \mathrm{II}] / \mathrm{H} \alpha$, intensity ratio increases with increasing distance from HII regions, whereas the corresponding increase of the $[\mathrm{N} \mathrm{II}] / \mathrm{H} \alpha$ ratio is less appreciable (see Fig. 4).

To identify the source of gas ionization, we constructed the diagnostic diagram showing the ratios of the fluxes of lines with different excitation mechanisms and similar wavelengths so that the result would not depend on dust extinction. Unfortunately, we can construct only one such diagram in the wavelength interval considered: $[\mathrm{N} \mathrm{II}] / \mathrm{H} \alpha$ versus $[\mathrm{S} \mathrm{II}] / \mathrm{H} \alpha$ (Fig 5 ). The dashed lines separate regions with different excitation mechanisms indicated as 'photoionization' and 'shock'. We assume, in accordance with Stasińska et al. (2006), that in the case of ionization by young stars the line rations follow to relations: $\log [\mathrm{S}$ II $] / \mathrm{H} \alpha<-0.4$, $\log [\mathrm{NII}] / \mathrm{H} \alpha<-0.4$. Higher relative intensity of forbidden lines means that shocks, or even a nonthermal source $(\mathrm{AGN})$, contribute appreciably to ionization. When inter-

1 Hereafter by the $[\mathrm{N} I \mathrm{II}] / \mathrm{H} \alpha$ and $[\mathrm{SII}] / \mathrm{H} \alpha$ ratios we mean the $[\mathrm{N} \mathrm{II}] \lambda 6583 / \mathrm{H} \alpha$ and $[\mathrm{S} \mathrm{II}](\lambda 6717+\lambda 6731) / \mathrm{H} \alpha$ line flux ratios, respectively. 
preting the positions of data points on the diagram one must bear in mind that: (1) boundaries of the photoionization domain are somewhat controversial. Namely, the boundaries are determined not only by particular models, but also by the statistics of observations of different galaxies samples; (2) in real objects the combined effect of several ionization sources is present. Thus, according to Stasińska et al. (2006), the $-0.2<\log [\mathrm{N} \mathrm{II}] / \mathrm{H} \alpha<-0.4$ domain corresponds to a composite source of ionization in LINER-type galaxies: photoionization by stars plus the effect of shocks, and shocks dominate only at $\log [\mathrm{NII}] / \mathrm{H} \alpha>-0.4$. Note that many researchers use even harder criterion (e.g., $\log [\mathrm{N} \mathrm{II}] / \mathrm{H} \alpha>0$ in Veilleux, Cecil \& Bland-Hawthorn 2005).

Figure 5 shows that the gas in the circumnuclear region (i.e. central part observed with the MPFS, and the $r<5$ arcsec regions in slit \#1 and $r<10$ arcsec regions in slit $\# 2$ ) is ionized by young stars. In the outer filaments shock ionization begins to dominate with increasing distance from the disc plane, according to the $[\mathrm{S} \mathrm{II}] / \mathrm{H} \alpha$ criterion. At the same time, the $[\mathrm{N} \mathrm{II}] / \mathrm{H} \alpha$ criterion indicates that all our measurements, except several data points with $r \approx 30 \operatorname{arcsec}$ in slit \#2, lie in the photoionization domain. However, in this case we consider the latter criterion to be less important, because the observed line ratio agrees well with the results of model computations Allen et al. (2008) for shock+precursor ionization with number density $n=1 \mathrm{~cm}^{-3}$ and solar metallicity. Figure 5 shows the grid of parameters for this model. It is evident from this figure that with increasing $r$ the observed data points lie along the lines of the increasing shock velocity from 200 to $300 \mathrm{~km} \mathrm{~s}^{-1}$ for small values of magnetic parameter $0-1 \mu G \mathrm{~cm}^{2 / 3}$. For estimation of gas mettalicity we used the data of slit \#2 that passes through the center of the brightest HII region, where the $[\mathrm{N} \mathrm{II}] / \mathrm{H} \alpha$ ratio is minimal and equal to $0.16 \pm 0.03$. If we assume that the shock contribution is minimal at this location then the approximation of Stasińska et al. (2006) implies a gas metallicity of $0.74 \pm 0.04 Z_{\odot}$, i.e. the solar-metallicity approximation is quite applicable to NGC 4460.

It thus follows that gas ionization in the extended $\mathrm{H} \alpha$ nebulosity can be explained by the combined effect of shocks and photoionization by young stars with the latter dominating in the inner regions. The galaxy lacks an active nucleus and therefore the likely cause of shocks is star formation. Gas is blown above the plane of the galaxy by the combined action of supernovae and stellar winds from young massive stars. This phenomenon is known as a 'galactic wind' (see a review by Veilleux et al. 2005). For comparison, we present in Fig. 5 the line ratios for three well studied galaxies where the existence of galactic wind has long been proven: M 82, NGC 253, and NGC 1569. For M 82 we give two average values obtained by Westmoquette et al. (2009), who used DensePak and GMOS instruments. We adopt the galactic-wind data for NGC 253 from Fig. 5 in Matsubayashi et al. (2009), and for NGC 1569 we give several estimates for external emission regions adopted from Fig. 7 in Heckman et al (1995), for slit $P A=70^{\circ}$ and $r=$ $\pm 20, \pm 50, \pm 70$ arcsec from the center. On the diagnostic diagram the published data for these well-known galactic winds scattered in relative wide range $( \pm 0.5 \mathrm{dex})$, which also includes the data points for NCC 4460 . Shock contribution to gas ionization in the filaments of NCC 4460 is comparable to galactic winds for all three galaxies hitherto studied. More-
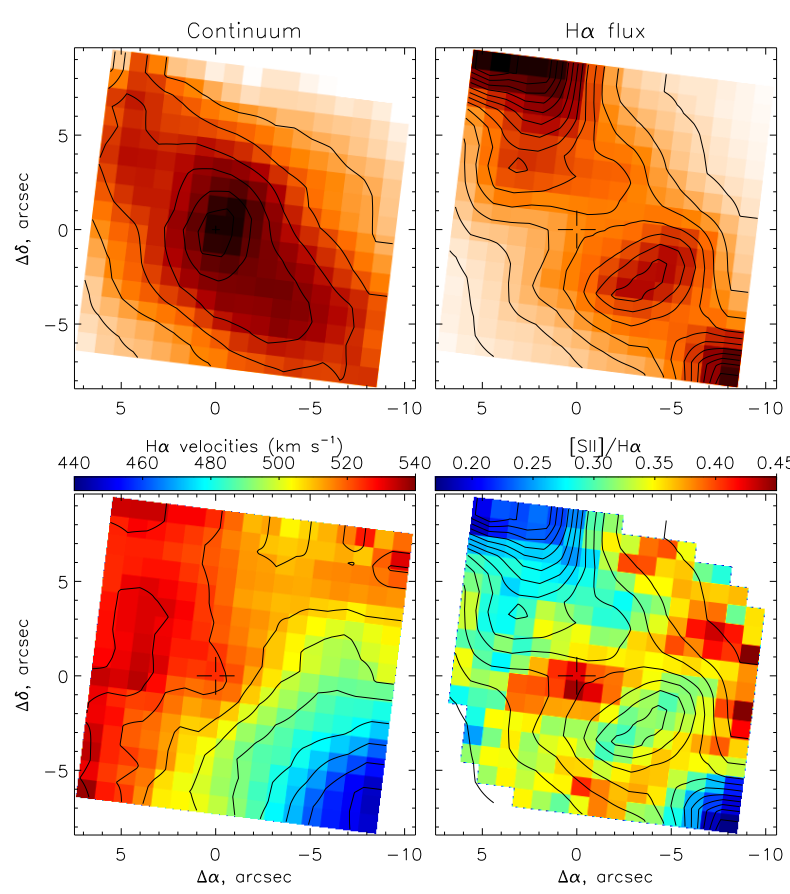

Figure 3. Results of MPFS observations. The top panel shows the continuum image taken near $\lambda 6400 \AA$ (left) and the $\mathrm{H} \alpha$ map (right). The bottom panel shows the $\mathrm{H} \alpha$ velocity field (left) and the map of the $[\mathrm{S} \mathrm{II}](\lambda 6717+\lambda 6731) / \mathrm{H} \alpha$ flux ratio with $\mathrm{H} \alpha$ contours superimposed (right). The coordinate origin coincides with the dynamic center (the cross).

over, the $[\mathrm{S} \mathrm{II}] / \mathrm{H} \alpha$ ratio in NGC 4460 is comparable with the values observed in NGC 253 and NGC 1569. That is not for $[\mathrm{N}$ II $] / \mathrm{H} \alpha$, because the intensity of the nitrogen doublet also depends from from metallicity. For instance, $[\mathrm{N}$ II $] / \mathrm{H} \alpha$ ratio in NGC253 is most likely due to the nitrogen overenrichment of the interstellar medium (Matsubavashi et al. 2009).

We use the $[\mathrm{S}$ II] line doublet ratio to estimate the electron density $n_{e}$ in accordance with the relation of Osterbrock (1989) for $T_{e}=10000 \mathrm{~K}$. Figure 6] shows the radial variations of of the line ratio and corresponded values of $n_{e}$. Unfortunately, Osterbrock's relation can be applied with confidence only at $n_{e}>50-100 \mathrm{~cm}^{-3}$, whereas at low densities the slope of the ([S II $] \lambda 6717 / \lambda 6731$ vs $\left.n_{e}\right)$ relation flattens and therefore leads to large uncertainties. Although the observed line ratio lies mainly inside this 'inconvenient' interval, it is safe to conclude that the density of emitting gas is low than $100 \mathrm{~cm}^{-3}$ in the disc of the galaxy. Outer filaments exhibit a large scatter of density values ranging from zero to $200-300 \mathrm{~cm}^{-3}$. It might be supposed that in the outer regions the spectrograph slit crosses individual clumps with highly nonuniform density distribution.

\section{THE GAS KINEMATICS}

The $\mathrm{H} \alpha$-line velocity field based on MPFS measurements (Fig. 3) can be described fairly well in terms of the model of circular rotation of the thin disc. The line-of-sight velocities measured by the $[\mathrm{NII}]$ and $[\mathrm{S} \mathrm{II}]$ lines show no appreciable deviations from $\mathrm{H} \alpha$ line-of-sight velocities. The center of 

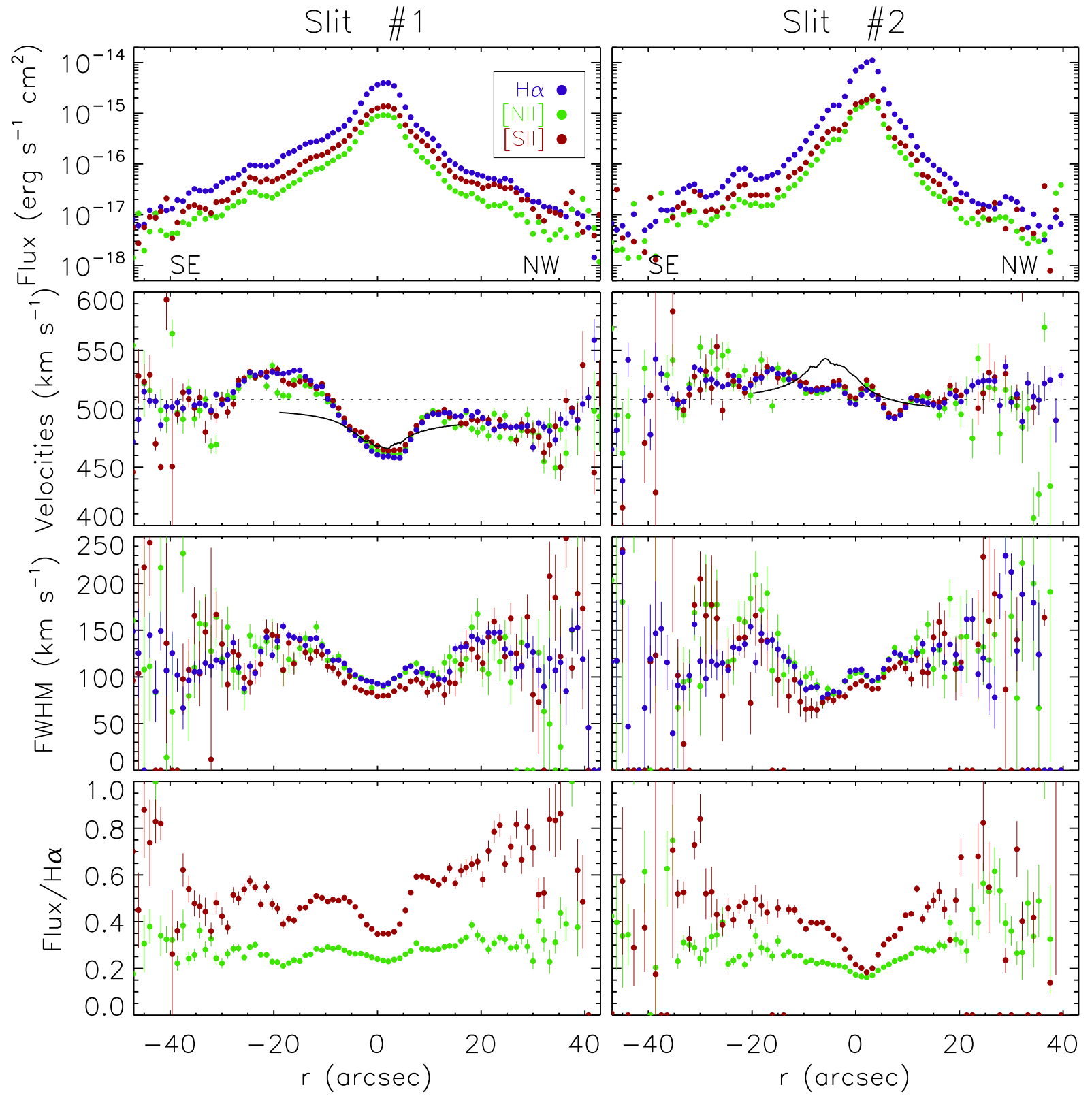

Figure 4. Measurements of parameters of the $\mathrm{H} \alpha,\left[\mathrm{N}_{\mathrm{II}}\right] \lambda 6583$ and $\left[\mathrm{S}_{\mathrm{II}}\right] \lambda 6717+6731$ emission lines along the slits \#1 (left) and \#2 (right). From top to bottom: surface brightness, line-of-sight velocity, FWHM (corrected for the widths of the instrumental profile), and the line-to-H $\alpha$ flux ratio. The dashed and solid lines show the systemic velocity (according to MPFS data) and the cross section of the model velocity field, respectively.

rotation defined as the symmetry center of the velocity field coincides with the center of the continuum image.

We constructed the model of rotation using the method described by Moiseev et al. (2004) with the position angle and inclination of the disc fixed in accordance with photometric data (Sect. 2.2). The $P A$ measured from the velocity field agrees with the photometric position angle within the errors. The systemic velocity is $V_{\text {sys }}=508 \pm \mathrm{km} \mathrm{s}^{-1}$. The rotation curve shows solid-rotation increase up to $v_{\max }=$ $67 \mathrm{~km} \mathrm{~s}^{-1}$ at $r=20$ arcsec that is a border of MPFS fieldof view. This value is close to the maximum rotation velocity according to HI measurements (Sage \& Welch 2006) Observed velocities deviate from the corresponding model values by up to $20 \mathrm{~km} \mathrm{~s}^{-1}$ along the minor axis of the galaxy, and this behavior is most probably indicative of gas ejection above the plane of the galaxy. It could also be due to the uncertainties in the model: the thin-disc approximation may perform poorly at $i=77^{\circ}$, internal absorption should be taken into account, etc.

The ionized gas velocity field in the compact star cluster region noted on optical images at $\sim 2$ arcsec North of the nucleus (see Sect. 2.1) does not show any kinematic peculiarities, like non-circular motions or shift of the rotation center. Therefore, our data do not support that this cluster is a nucleus of a dwarf satellite - a merging remnant. 


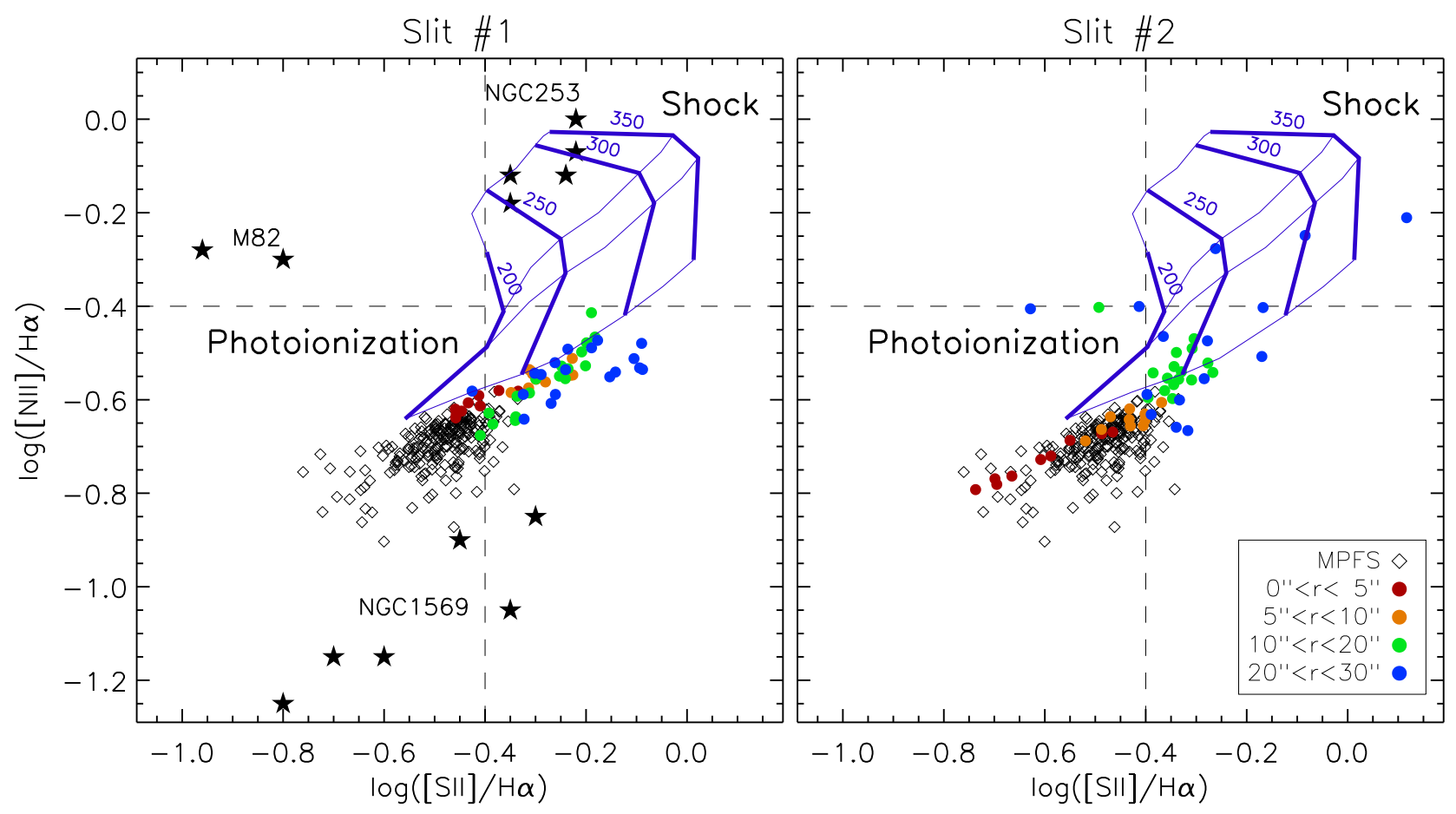

Figure 5. The diagram of the $[\mathrm{N} \mathrm{II}] / \mathrm{H} \alpha$ vs $[\mathrm{S} \mathrm{II}] / \mathrm{H} \alpha$ flux ratios. The dashed line separates domains with different ionization mechanisms. The blue lines show the grid of shock+precursor ionization models according to Allen et al. (2008) for $n=1 \mathrm{~cm}^{-3}$ and solar elemental abundances. The thin and bold blue lines mark the contours of constant magnetic parameter $0.001,0.5,1,5 \mu G \mathrm{~cm}^{2 / 3}$ (from bottom to top) and contours of constant shock velocity (which is labeled in $\mathrm{km} \mathrm{s}^{-1}$ ), respectively. The circles show the measurements made along slits \#1 (left) and \#2 (right). Different colors correspond to different distances along the slits. The diamond signs indicate the results of MPFS measurements for the circumnuclear region. The asterisks in the left-hand figure show the measurements for the regions of galactic wind in M 82 (Westmoquette et al. 2009), NGC 253 (Matsubayashi et al. 2009), and NGC 1569 (Heckman et al 1995).

New high-resolution stellar kinematics data are needed for understanding the origin of this off-set cluster.

To estimate the peculiar velocities of the outer filaments, we extrapolate our model out to greater distances assuming that the rotation curve reaches a plateau. Figure 4 compares the model extrapolation and observed velocities in slit cross sections. Slit \#1 shows a good agreement between the model and observations at $r<15$ arcsec in the NW half of the nebulosity, whereas its more extended SE half exhibits appreciable deviations already beyond $7-10$ arcsec from the center with the discrepancies amounting to $30-35 \mathrm{~km} \mathrm{~s}^{-1}$. Slit \#2 passes farther from the center, along the edge of the field of view of the MPFS, and therefore here deviations from the model extrapolation are more conspicuous in the disc region. On the whole, we may conclude that in the outer filaments systematic deviations of the observed line-of-sight velocities from the corresponding model values do not exceed, on the average, $30 \mathrm{~km} \mathrm{~s}^{-1}$. This implies an outflow velocity of $v_{\text {out }} \approx 130 \mathrm{~km} \mathrm{~s}^{-1}$, if we assume that the observed peculiar gas motions are directed perpendicularly to the plane of the disc and take projection effect into account. This value is comparable to the observed halfwidth of emission lines, which amounts to $150-170 \mathrm{~km} \mathrm{~s}^{-1}$ at a distance of $r=20-30 \operatorname{arcsec}(0.9-1.4 \mathrm{kpc})$, whereas the FWHM is half in the disc. The fact that the amplitudes of regular velocities are approximately equal to those of chaotic velocities are indicative of strong turbulence in the filament gas.

\section{DISCUSSION}

\subsection{Galactic wind}

We showed above that the peculiarities of the distribution of the parameters of ionized gas (kinematics, density, and state of ionization) observed in NGC 4460 can be most easily explained in terms of the 'galactic wind' hypothesis, i.e., assuming gas outflow above the plane of the galaxy produced by a central burst of star formation. The alternative assumption suggesting that we observe remnants of a tidally disrupted gas-rich companion is ruled out, because it would imply that line-of-sight velocities in filaments should deviate significantly from the systemic velocity. Besides the above indicators, the shape of the emission nebulosity also suggests the presence of galactic wind. The nebulosity resembles a butterfly or, rather, two bubbles expanding above the plane of the galaxy. Note that the diameter of the bubble has become greater than the halfwidth of the gaseous disc, it has open vertex, because the latter is no longer compressed by the ambient gas. Moreover, the emission nebulosity resembles the well-known superwind galaxy NGC 1482 (see review by Veilleux et al. 2005, for this and other examples). The nonuniform distribution of the density in the emission nebulosity is not surprising, because the optical emission lines are thought to originate from a boundary between hot bubble gas and cool ambient ISM, fragmented under the action of Kelvin-Helmholtz and Rayleigh-Taylor instabilities.

Regions of modern star formation are located at the 
base of the wind and we showed that ongoing star formation is concentrated almost entirely inside a ring less than $2 \mathrm{kpc}$ in diameter. Gas is ionized not only by the radiation of OB stars, but also by shocks produced by the combined effect of supernovae and winds of massive stars, which transfer kinetic energy to the interstellar medium. The observed lineof-sight velocities in filaments are small and close to the systemic velocity, suggesting that these features consist of gas that was ejected from central regions and has preserved its angular momentum. The gas outflow occurs mostly in the sky plane and therefore the line-of-sight projection of the outflow velocity is small.

We adopt the characteristic length of the brightest filaments $l=30-35 \operatorname{arcsec}(1.3-1.6 \mathrm{kpc})$ to derive the dynamic age of the emission feature $\tau_{\text {dyn }}=l / v_{\text {out }} \approx 10-$ $12 \mathrm{Myr}$. The total $\mathrm{H} \alpha$ luminosity of the galaxy is $L_{H \alpha}=$ $1.7 \times 10^{40} \mathrm{erg} \mathrm{s}^{-1}$ based on the observed $\mathrm{H} \alpha+\left[\mathrm{N}_{\mathrm{II}}\right]$ flux from Kaisin \& Karachentsev (2008) and the average line ratio of $\log [\mathrm{N} \mathrm{II}] / \mathrm{H}=-0.7$. We assume that the wind radiation includes the flux from the regions located outside the ellipse with the semi-major axis 25 arcsec and axial ratio 0.4 , which includes all HII regions in the disc. The wind luminosity is $L_{H \alpha}($ wind $)=2.7 \times 10^{39} \mathrm{erg} \mathrm{s}^{-1}$, it makes up for 16 per cent of the total $L_{H \alpha}$, and the SE and NW parts of the bipolar structure have almost equal luminosities ( 9 and 7 per cent, respectively). We now adopt, in accordance with Fig. 6] a mean electron density of $n_{e}=50 \mathrm{~cm}^{-3}$ to infer, in the same way as Matsubayashi et al. (2009), the total mass of ionized gas ejected from the dis屯 $2, M_{\text {wind }}=1.7 \times 10^{5} M_{\odot}$, and its kinetic energy $E=5.8 \times 10^{52}$. We describe the emitting volume of the SE part of the outflow wind by a truncated cone with the bases of diameters 30 and 40 arcsec to derive a characteristic filling factor for this volume $f \approx 3 \times 10^{-5}$. This value of $f$ is indicative of the highly nonuniform nature of the emitting medium - $\mathrm{H} \alpha$ emitting gas is located mostly in the walls of the bubble, which fragment under the action of characteristic instabilities (Veilleux et al. 2005).

We now compare our inferred parameters with the wind parameters in two well-known nearby galaxies: M82 (Shopbell \& Bland-Hawthorn 1998) and NGC 253 (Matsubayashi et al. 2009). The mass of the ejected gas in NGC 253 is almost the same as in our case, although the kinetic energy of the gas is twice lower, whereas the formation time scale of the feature is almost six times shorter. In M82 $M_{w i n d}$ and $E$ are greater by almost a factor of 30 and 360, respectively, with closer $\tau_{d y n}=3.4 \mathrm{Myr}$. The wind outflow velocities in these galaxies are three to four times higher than in NGC 4460. Given our remark about the uncertainty of measured $v_{\text {out }}$, it can be concluded that, on the whole, the wind parameters in NGC 4460 are close to those observed in NGC 253, except for the fact that the emission nebulosity in NGC 4460 took much longer time to form. This is not surprising, because the total SFR formally inferred from $L_{H \alpha}$ is almost 10 times higher in NGC 253. This fact most likely

${ }^{2}$ In this analysis we used information about single phase of the wind, because $\mathrm{H} \alpha$ emission traces only the cooler interaction zone between hot wind fluid and the ambient medium. Therefore the values of $M_{\text {wind }}$ and $E$ are underestimated. However, we present its for comparison with the similar values taken from similar analyses in other galaxies.

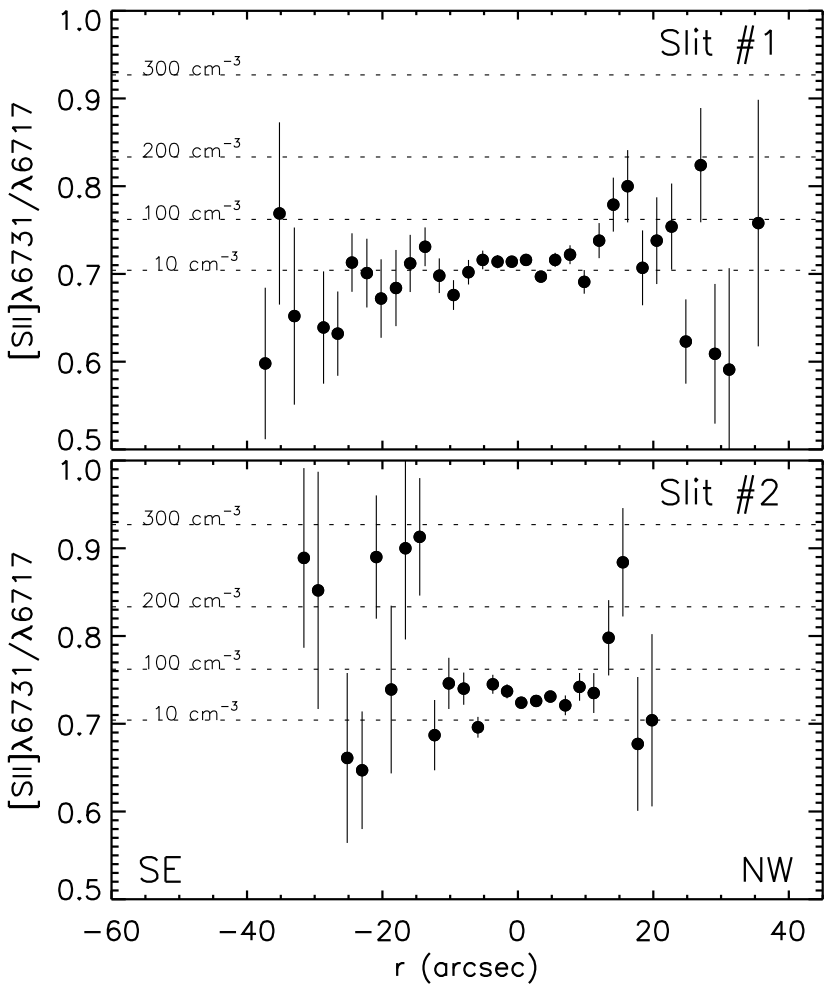

Figure 6. Variation of the $[\mathrm{S} \mathrm{II}]$ line doublet ratio for slit \#1 (top) and slit \#2 (bottom). The horizontal dashed lines indicate the values of electron density corresponded which are labeled near the lines.

determines the relatively lower outflow velocity. In particular, we do not know whether the kinetic energy of the wind is sufficient for the gas to escape from the galaxy. A simple estimate of the escape velocity obtained in terms of the isothermal sphere model with a cutoff radius of 2 arcmin yields $v_{\text {esc }} \geq 2.7 v_{\max }=180 \mathrm{kms}^{-1}$ for regions located at galactocentric distances $r<8$ arcsec, which is appreciably greater than $v_{\text {out }}$. Therefore, most probably, after cooling down, the gas of the wind will fall back onto the galactic plane.

\subsection{Cause of the burst of star formation}

Thus the central kiloparsec in NGC4460 is engulfed in on-going star formation $\left(S F R=0.3 M_{\odot} / y r\right.$, see Kaisin \& Karachentsev 2008), in contrast to the rather old and 'quiet' disc of the galaxy. What could have triggered star formation in this lenticular galaxy? Tidal interaction with a companion is unlikely since the galaxy appears isolated. Neither there is any evidence for a merger of a gas-rich dwarf companion - the velocity field lacks kinematically decoupled components to be expected in such a case. No tidal features can be seen on optical images or published low-resolution HI maps (Sage \& Welch 2006). The inner disc that we found in the brightness distribution proves that star formation has been going on for quite a long time in the center, however it is not indicative of interaction.

We already pointed out above that isolated E and S0 galaxies are rather rare objects, their integrated parameters differ appreciably from normal $\mathrm{E}$ and $\mathrm{S} 0$ galaxies in 
groups and clusters. Table 2 lists the basic parameters of all the three representatives of this category of galaxies found within $10 \mathrm{Mpc}$ from the Sun. Row (2) gives the tidal index according to Karachentsev et al. (2004); distances in row (3) are from Tonrv et al. (2001) for except NGC 404 for which mean value from Tonry et al. (2001) and Karachentsev et al. (2002) is accepted; Rows (1), (4) and (5) give the observed parameters of the galaxies adopted from the NED database; Row (6) presents the HI mass from HyperLEDA database adopted for the accepted distances. Row (7) gives the estimated global star-formation rate in galaxies inferred from their $\mathrm{H} \alpha$ fluxes from Kaisin \& Karachentsev (2008) for NGC 4460 and from Karachentsev \& Kaisin (2010) for other two galaxies. The last rows give two dimensionless and distance independent parameters (see Karachentsev \& Kaisin (2007) and Kaisin \& Karachentsev (2008) for details): $P_{K}^{*}=$ $\log \left([S F R] \times T_{0} / L_{K}\right)$ and $F^{*}=\log \left(M_{H I} /[S F R] \times T_{0}\right)$. The former characterizes the proportion of its luminosity the galaxy would produce during the Hubble time $T_{0}=13.7$ Gyr at the current rate of star formation and the mass-to(K-band)-luminosity ratio $M / L_{K}=1 M_{\odot} / L_{\odot}$. The parameter $F^{*}$ shows how much Hubble time the galaxy will need in future to spend the present supply of gas if star formation proceeds at the currently observed rate.

Distribution of 420 Local volume galaxies in the 'pastfuture' diagram (Karachentsev \& Kaisin 2010) is presented in Fig. 7 The discussed isolated early-type galaxies of moderate luminosity are distinguished on the diagram by red color. Here, galaxies of different morphological types occupy essentially different regions in the diagnostic diagram. The median values of $P_{K}^{*}$ and $F^{*}$ for the total $\mathrm{LV}$ sample are -0.40 and -0.25 , respectively. These quantities mean that a typical LV galaxy requires $2-3$ times higher SFR in the past to reproduce its observed luminosity, having also enough amount of gas to continue star formation with the observed rate during the next 8 Myrs.

The NGC 404, NGC 855, and NGC 4460 galaxies differ appreciably in luminosity, but have almost similar hydrogen masses $\sim 1 \times 10^{8} M_{\odot}$. Specific star-formation rate per unit luminosity differs little among these galaxies. The parameter $P_{K}^{*}$ is only slightly smaller than zero for all three galaxies, and this indicates that the galaxies are capable of reproducing most of their stellar mass during cosmological time $T_{0}$ provided that star formation is at the current level. At the same time, the available gas reserves in these galaxies can maintain the current rate of star formation only for a short time scale ranging from $1 / 40$ (NGC 4460) to $1 / 6$ (NGC 404) of the age of the Universe.

The location of isolated E and S0 galaxies on the diagnostic diagram $\left\{P_{K}^{*}, F^{*}\right\}$ does not allow us to view their observed evolutionary stage as a burst of star formation triggered by a merger of a companion or a single hydrogen cloud. Should this be the case, one would expect in the Local Volume, in addition to the three isolated emission E and S0 galaxies mentioned above, an order of magnitude greater number of similar objects at the quiescent interburst stage. However, the currently available $\mathrm{H} \alpha$-flux data for 420 galaxies of the Local Volume Karachentsev \& Kaisin (2010) do not support the existence of such a population. Besides NGC 4460, NGC 404, and NGC 855, the Local Volume contains only two other isolated galaxies: NGC 2787 (S0-a) and NGC 4600 (S0) where very weak $\mathrm{H} \alpha$ emission is presented.
Table 2. Integrated parameters of isolated E/S0 galaxies within $10 \mathrm{Mpc}$

\begin{tabular}{rlrrr}
\hline \hline Line & Parameter & NGC 4460 & NGC 855 & NGC 404 \\
\hline$(1)$ & Type & SB(s)0+ & $\mathrm{E}$ & SA(s)0- \\
$(2)$ & TI & -0.7 & -0.8 & -1.0 \\
$(3)$ & Distance $(\mathrm{Mpc})$ & 9.59 & 9.73 & 3.24 \\
$(4)$ & $\mathrm{M}_{B}(\mathrm{mag})$ & -17.89 & -17.07 & -16.61 \\
$(5)$ & $\mathrm{M}_{K}(\mathrm{mag})$ & -20.87 & -20.15 & -19.00 \\
$(6)$ & $\log \mathrm{M}(\mathrm{HI})(\mathrm{sun})$ & 7.92 & 8.13 & 7.97 \\
$(7)$ & $\log [\mathrm{SFR}]\left(\mathrm{M}_{\odot} / \mathrm{yr}\right)$ & -0.59 & -1.07 & -1.37 \\
$(8)$ & $P_{K}^{*}$ & -0.11 & -0.30 & -0.14 \\
$(9)$ & $F^{*}$ & -1.63 & -0.94 & -0.80 \\
\hline
\end{tabular}

These galaxies gave blue absolute magnitudes -18.5 and -15.7 , respectively.

The predominance of objects with current central star formation among isolated $\mathrm{E}$ and $\mathrm{S} 0$ galaxies and their capability of reproducing the available stellar mass over the cosmological time scale with the observed SFR combined with the scarcity of available gas reserves $\left(\sim 1 \times 10^{8} M_{\odot}\right)$ leads us to suggest that ongoing star formation in this galaxies is fed by an external source, i.e. intergalactic gas clouds or filaments. The process of external gas accretion should be have a regular steady character on a cosmological time scale, without significant bursts.

Recently Kannappan et al. (2009) described a population of 'blue-sequence E/S0s' resided on the locus of latetype galaxies in color vs. stellar mass parameter space. They argue that these galaxies actively (re)growing their stellar discs and therefore may form a bridge between late-type spirals/iregulars and early-type red-sequence galaxies. Many of low-to-intermediate mass blue-sequence $\mathrm{E} / \mathrm{S} 0 \mathrm{~s}$ reside in low density environments, often have a blue center and substantial fraction of cold gas. However their gas consumption time usually smaller than 3 Gyr (Wei et al. 2009). All these properties are similar with the Local Volume isolated E/S0 galaxies described above, therefore NGC 4460, NGC 404, and NGC 855 seem to be belonging to the population of blue-sequence E/S0s. Kannappan et al. (2009) suggest these galactic population connects with the disc rebuilding after major/minor merging or companions interactions. In the present paper we stress that accretion of external gas is also important in a particular case of the evolution of isolated early-type galaxies.

\section{SUMMARY}

We used available UV and optical images to explore the structure of the isolated lenticular galaxy NGC 4460. Also we obtained long-slit and 3D spectroscopic data to probe the origin of the bright $\mathrm{H} \alpha$ nebulosity surrounding the central region of the galaxy. We consider the following points to be the most important.

(i) All ongoing star formation with the rate about $0.3 M_{\odot} / y r$ resides in a pseudo-ring with radius about one kiloparsec. Together with antitruncated shape of the surface brightness profile it suggests a recent formation of the inner exponential disc, whose radial scale three times smaller than global old stellar disc. 


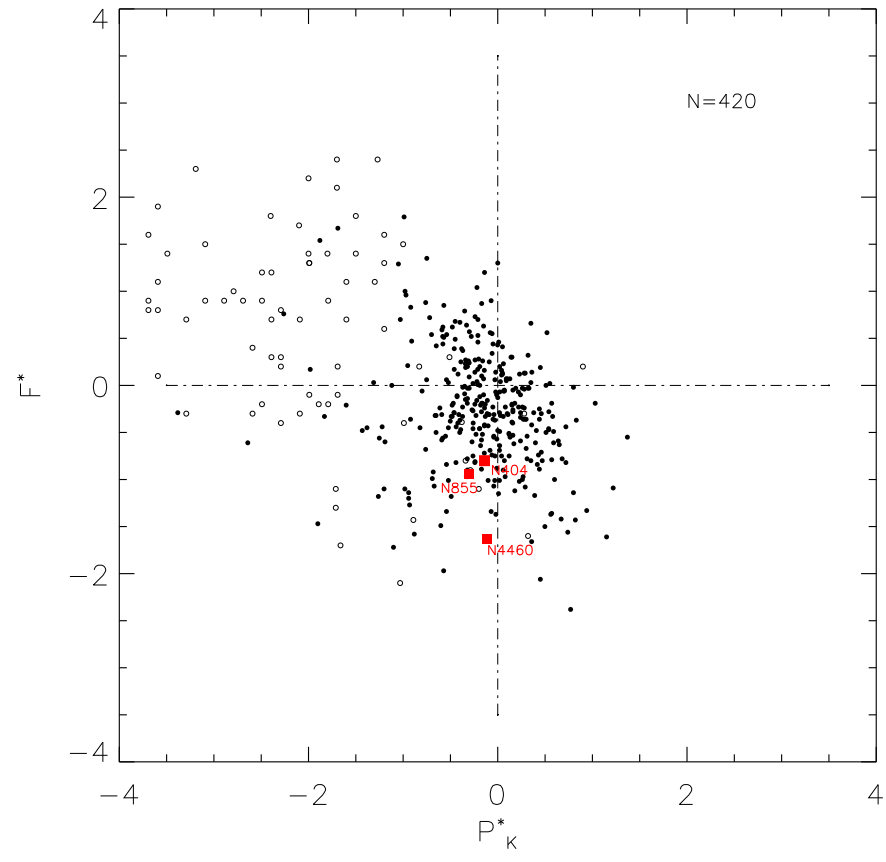

Figure 7. Evolutional plane 'past-future' for 420 Local Volume galaxies Karachentsev \& Kaisin 2010). The galaxies observed and detected in $\mathrm{H} \alpha$ are shown by filled symbols, and galaxies with only upper limit of their $\mathrm{H} \alpha$ flux are indicated by open circles. The isolated E/S0 galaxies of moderate luminosity are distinguished by red squares and marked with their NGC numbers.

(ii) Whereas gas in the circumnuclear disc is photoionized by radiation of young stars, the external regions of $\mathrm{H} \alpha$ nebulosity are ionized by shocks. This fact, in combination with a bi-conical shape of the ionized gas nebulosity, with the radial distributions of the emission lines velocities and width can be explained as a galactic-scale outflow (superwind) induced by a central star formation. We estimated outflow velocity as $\geq 130 \mathrm{~km} \mathrm{~s}^{-1}$. Galactic wind parameters, such as outflow velocity, formation time and total kinetic energy are several times smaller comparing with the known galactic wind in NGC 253, which is explained by substantially different star formation rates.

(iii) Also we discussed the evolutional status of NGC 4460 together with other well-isolated E/S0 galaxies in the local volume: NGC 404, and NGC 855. We considered the position of the galaxies on the 'past-future' diagram (Karachentsev \& Kaisin 2010) and suggested that continuous accretions of an intergalactic gas is most probable source of their current star formation. In contrast to merging scenario, the external gas accretion seems to be a sluggish process without violent events.

The research is partly based on data obtained from the Multimission Archive at the Space Telescope Science Institute (MAST). STScI is operated by the Association of Universities for Research in Astronomy, Inc., under NASA contract NAS5-26555. Support for MAST for non-HST data is provided by the NASA Office of Space Science via grant NAG5-7584 and by other grants and contracts. Funding for the SDSS and SDSS-II has been provided by the Alfred P. Sloan Foundation, the Participating Institutions, the National Science Foundation, the US Department of
Energy, the National Aeronautics and Space Administration, the Japanese Monbukagakusho, the Max Planck Society and the Higher Education Funding Council for England. The SDSS web site is http://www.sdss.org/. This research has made use of the NASA/IPAC Extragalactic Database (NED) which is operated by the Jet Propulsion Laboratory, California Institute of Technology, under contract with the National Aeronautics and Space Administration. We acknowledge the usage of the HyperLeda database (http://leda.univ-lyon1.fr). This work was supported by the Russian Foundation for Basic Research (projects no. 09-0200870, no. 07-02-00005, and no. 08-02-00627). AM is also grateful to 'Dynasty' Fund. The authors thank the anonymous referee for his constructive advice that helped us to improve the paper.

\section{REFERENCES}

Afanasiev V. L., Dodonov S. N., Moiseev A. V., 2001, in Ossipkov L. P., Nikiforov I. I., eds, Stellar Dynamics: from Classic to Modern, Saint Petersburg, p. 103

Afanasiev V. L.,\& Moiseev A. V., 2005, Astronomy Letters, 31, 193

Allen M .G., Groves B .A., Dopita M .A., Sutherland R .S., Kewley L .J., 2008, ApJS, 178, 20

Del Rio M. S., Brinks E., Cepa J. 2004, AJ, 128, 89

Erwin P., Beckman J. E., Pohlen M., 2005, ApJ, 626, L81

García-Barreto J.A., Franco J., Carrillo, R., Venegas S., Escalante-Ramírez B., 1996, RMxAA, 32, 89

Heckman T. M., Dahlem M., Lehnert M. D., Fabbiano G., Gilmore D., Waller W. H., 1995, ApJ, 448, 98

Hubble E. P., 1926, ApJ, 64, 321

Kaisin S. S.,\& Karachentsev I. D., 2008, A\& A, 479, 603

Kannappan S J., Guie J. M., Baker A. J. 2009, AJ, 138, 579

Karachentsev I. D., Kaisin S. S., 2007, AJ, 133, 1883

Karachentsev I. D., Kaisin S.S., 2010, in preparation

Karachentsev I. D.., Sharina M. E., Makarov D. I., et al. 2002, A\&A, 389, 812

Karachentsev I. D.., Karachentseva V. E., Huchtmeier W. K., Makarov D. I. 2004, AJ, 127, 2031

Karachentsev I. D.., Makarov D. I., Karachentseva V.E., Melnyk O.V. 2009, Proceedings conference "Galaxies in Isolation", Granada, in press

Koopmann R. A., Kenney J. D., 2006, ApJS, 162, 97

Kormendy J., Kennicutt R. C., 2004, ARA\&A, 42, 603

Matsubayashi K., Sugai H., Hattori T., Kawai A., Ozaki S., Kosugi G., Ishigaki T., Shimono A., 2009, ApJ, 701, 1636

Moiseev A. V., Valdés J. R., Chavushyan V. H. 2004, A\&A, 421,433

Osterbrock, D. E. 1989, Astrophysics of Gaseous Nebulae and Active Galactic Nuclei. University Science Books, Mill Valley, CA

Sage , L. J., Welch G.A. 2006, ApJ, 644, 850

Shopbell P. L., Bland-Hawthorn J. 1998, ApJ, 493, 129

Stasińska G., Cid Fernandes R., Mateus A., Sodré L., Asari N. V., 2006, MNRAS, 371, 972

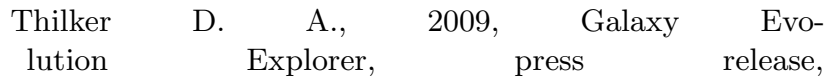
http://www.galex.caltech.edu/newsroom/glx2008-02r.html 
Tonry J. L., Dressler A., Blakeslee J. P., et al. 2001, ApJ, 546,681

Veilleux S., Cecil G., Bland-Hawthorn J. 2005, ARA\&A, 43, 769

Wei L. H., Kannappan S J., Vogel S. N., Baker A. J. 2009, ApJ, accepted, arXiv:0911.2248 1 [astro-ph.CO]

Westmoquette M. S., Gallagher J. S., Smith L. J., Trancho G., Bastian N., Konstantopoulos I. S., 2009, ApJ, 706, 1571 